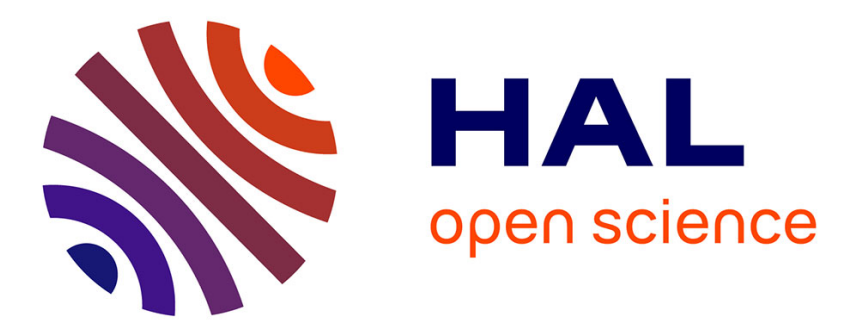

\title{
Partial genotyping at polymorphic markers can improve heritability estimates in sibling groups
}

Julie Gaüzere, Sylvie Muratorio, Laurène Gay, Etienne K. Klein

\section{To cite this version:}

Julie Gaüzere, Sylvie Muratorio, Laurène Gay, Etienne K. Klein. Partial genotyping at polymorphic markers can improve heritability estimates in sibling groups. Molecular Ecology Resources, 2016, 16 (6), pp.1340-1352. 10.1111/1755-0998.12536 . hal-01509032

\section{HAL Id: hal-01509032 \\ https://hal.science/hal-01509032}

Submitted on 15 Apr 2017

HAL is a multi-disciplinary open access archive for the deposit and dissemination of scientific research documents, whether they are published or not. The documents may come from teaching and research institutions in France or abroad, or from public or private research centers.
L'archive ouverte pluridisciplinaire HAL, est destinée au dépôt et à la diffusion de documents scientifiques de niveau recherche, publiés ou non, émanant des établissements d'enseignement et de recherche français ou étrangers, des laboratoires publics ou privés. 
Title: Partial genotyping at polymorphic markers can improve heritability estimates in sibling

$$
\text { groups }
$$

Authors: Gauzere J. ${ }^{1,2}$, Oddou-Muratorio S. ${ }^{2}$, Gay L. ${ }^{3}$, Klein E. K. ${ }^{2,4, *}$

\section{Adresses:}

${ }^{1}$ CEFE, CNRS, 34000 Montpellier, France

${ }^{2}$ URFM, INRA, 84000 Avignon, France

${ }^{3}$ AGAP, INRA, 34000 Montpellier, France

${ }^{4}$ BioSP, INRA, 84000 Avignon, France

Key words: Heritability; Pedigree-free methods ; Marker-based relatednesses ; Unequal reproductive success; Maternal Effects; Inbreeding

\section{Corresponding author:}

Etienne Klein

INRA, Biostatistique et Processus Spatiaux

Domaine Saint Paul, Site Agroparc

84914 Avignon Cedex 9, France

Fax: +33(0)4 32722902

etienne.klein@avignon.inra.fr

Running title: Improving $h^{2}$ estimates in sibling designs

This article has been accepted for publication and undergone full peer review but has not been through the copyediting, typesetting, pagination and proofreading process, which may lead to differences between this version and the Version of Record. Please cite this article as doi:

$10.1111 / 1755-0998.12536$

This article is protected by copyright. All rights reserved. 


\section{Abstract}

Accurate estimates of heritability $\left(h^{2}\right)$ are necessary to assess adaptive responses of populations and evolution of fitness-related traits in changing environments. For plants, $h^{2}$ estimates generally rely on maternal progeny designs, assuming that offspring are either half-sibs or unrelated. However, plant mating systems often depart from half-sib assumptions, this can bias $h^{2}$ estimates. Here, we investigate how to accurately estimate $h^{2}$ in non-model species through the analysis of sibling designs with a moderate genotyping effort.

We performed simulations to investigate how microsatellite marker information available for only a subset of offspring can improve $h^{2}$ estimates based on maternal progeny designs in presence of non-random mating, inbreeding in the parental population or maternal effects. We compared the basic family method, considering or not adjustments based on average relatedness coefficients, and methods based on the animal model. The animal model was used with average relatedness information, or with hybrid relatedness information: associating one-generation pedigree and family assumptions, or associating one-generation pedigree and average relatedness coefficients.

Our results highlighted that methods using marker-based relatedness coefficients performed as well as pedigree-based methods in presence of non-random mating (i.e. unequal male reproductive contributions, selfing), offering promising prospects to investigate in situ heritabilities in natural populations. In presence of maternal effects, only the use of pairwise relatednesses through pedigree information improved the accuracy of $h^{2}$ estimates. In that case the amount of father-related offspring in the sibling design is the most critical. Overall, we showed that the method using both one-generation pedigree and average relatedness coefficients was the most robust to various ecological scenarios.

This article is protected by copyright. All rights reserved. 


\section{Introduction}

In a population, significant additive genetic variance $\left(V_{A}\right)$ is required for traits to evolve in response to guided selection in livestock or crops and to environmental pressure in natural populations (Hill \& Kirkpatrick, 2010). The additive genetic variance scaled by the phenotypic variance, the so-called heritability $\left(h^{2}\right)$, summarizes the proportion of variance due to additive genetic effects and provides a good proxy of the ability of populations to evolve (Pigliucci, 2008). Compared to the level of genetic variance between populations, $V_{A}$ also provides an index to evaluate the adaptive divergence or convergence of traits (Qst; Merilä \& Crnokrak 2001). All the classical methods that estimate $V_{A}$ compare in some way the phenotypic and genetic resemblances between pairs of relatives. They thus rely on the knowledge or the estimation of genetic relatedness ( $\rho$; Falconer \& MacKay 1996), which is often critical for studies in natural populations.

The traditional and easiest way to conduct quantitative genetic analysis is to use designs with a priori knowledge about the relatedness relationships among individuals. Because of the fundamental divergence in their life history traits (e.g. mobility, population sizes, fecundities), plant and animal quantitative genetics have developed different methodologies, in terms of both sampling strategies and statistical models. In plants, the large number of seeds produced per mother from one reproductive event favored the sampling of maternal open-pollinated (OP) families. It enabled large trials (over 1000 offspring ; e.g. El-Kassaby et al. 2011), which efficiently reduced the estimation error of quantitative genetic parameters. The OP families are classically analysed with the "family model" (Falconer \& MacKay, 1996), assuming homogeneous and exact half-sib (HS) relationships between offspring from the same family. In animals

This article is protected by copyright. All rights reserved. 
(e.g. mammals and birds), the easier observation of matings, the lower population sizes and fecundities, promoted long-term population monitoring and inference of parent-offspring relationships over several generations. The heterogeneous relatedness structure, summed up in multi-generational pedigrees, are analysed with the "animal model" (Henderson, 1984; Kruuk, 2004). A practical issue, common to plants and animals, is to improve the relatedness information injected in the analysis, to avoid observation errors in pedigrees and to inform more precisely the relatedness relationships within maternal progenies.

Despite these methodological divergences, we expect an important benefit from applying the animal model to plant species because, $(i)$ the use of pedigree information through an animal model has proved its efficiency in animal natural populations (Kruuk, 2004) and (ii) the relatedness structure in most plant natural populations often departs from family model assumptions (Gauzere et al., 2013b). Treating samples within maternal families as "real" HS indeed supposes that (1) matings occur with many equally fecund males and without preferential mating, (2) mates are unrelated and there is no self-fertilization and (3) mothers are not inbred (Gauzere et al., 2013b). Moreover, the family model (4) is not suited to account for environmental effects confounded with the family structure, such as environmental parental effects. However, most plant species present mixed-mating systems (i.e. both outcrossing and selfing; Ward et al. 2005), unequal reproductive success (Kang et al., 2003), significant spatial genetic structure (Vekemans \& Hardy, 2004) and distance-limited pollen dispersal leading to preferential mating (Ashley, 2010). Finally, maternal effects are frequently reported for seedling's traits (Roach \& Wulff, 1987).

This article is protected by copyright. All rights reserved. 
For more than 40 years, plant quantitative genetics was particularly creative in new methodologies that account for departure from HS assumptions. This is especially true for trees, for which controlled cross experiments are difficult. The pioneer study from Squillace (1974) proposed to adjust the $V_{A}$ and $h^{2}$ estimates from the family model according to the average relatedness between offspring within OP families expected from the empirical knowledge about the mating system of the species. This approach is still widely used for trees to account for inflated relatednesses within families (e.g. Ramirez-Valiente et al. 2009). However, it does not account for the between-families variability of mating characteristics, which is often significant (e.g. Gauzere et al. 2013a).

The development of polymorphic markers, such as microsatellites, has offered the opportunity to refine relatedness information and to use individual-based quantitative genetic models (i.e. animal model) in non-model species. First, molecular information was used to improve pedigrees thanks to parentage assignment (Marshall et al., 1998; Hadfield et al., 2006) and sibship/pedigree reconstruction methods (Blouin, 2003; Fernandez \& Toro, 2006; Cros et al., 2014). Nevertheless, in plants, pedigrees can generally be only reconstructed for a subset of the phenotyped offspring and over one-generation, for both natural populations (Stinchcombe, 2014) and some breed populations (notably in trees; El-Kassaby et al. 2011). The exhaustive genotyping of the offspring and parental populations is indeed a strong experimental constraint since (i) traditional plant family designs are large and (ii) the high pollen immigration rates and large population sizes prevent the identification of an exhaustive but "reasonable" set of potential mates. This observation conducted El-Kassaby et al. (2011) to propose the "Breeding without breeding" concept to improve the estimation of $V_{A}, h^{2}$ and the breeding values in OP 
designs. This approach uses a subset of offspring with one-generation pedigree information (i.e. half- and full-sibs relationships; HS, FS), reconstructed from molecular data, combined with the assumption of half-sibships (HS) for individuals lacking genotypes. This study showed that a modest genotyping effort can effectively improve quantitative genetics estimates.

Alternatively, the problem of getting a complete and accurate genealogy can be circumvented by directly estimating the relatedness matrix $(A)$ from molecular markers and bypassing pedigrees (Frentiu et al., 2008; Gay et al., 2013; Robinson et al., 2013). These pedigree-free approaches are limited by the ability of molecular markers to efficiently catch the relatedness variance of populations (Gay et al., 2013). Moreover, these approches still require the estimation of the whole matrix of pairwise relatednesses and thus the exhaustive genotyping of the offspring. Recently, Bush et al. (2011) proposed to modify the $A$ matrix by accounting for variable levels of inbreeding $(f)$ derived from selfing estimates at the family level. This approach nicely extends the adjustment from Squillace (1974), through an animal model procedure, to account for the variation of mating characteristics between families. By using average relatedness information at the family level, this approach seems particularly relevant to sibling designs where only a subset of the phenotyped offspring are genotyped. However, it neglects the inter-individual variability of relatedness within and between families, even for the genotyped individuals for which this information can be assessed.

Here, we investigated whether an optimal account of the whole relatedness information available could be to use both the reconstructed pedigree for the genotyped individuals and the family average relatedness coefficients for the non-genotyped individuals (instead of the family

This article is protected by copyright. All rights reserved. 
assumptions). In addition to proposing new methodological developments, our goal is to properly compare the existing approaches presented above, which exploit relatedness information at different levels (i.e. population, family, individual). The classical view is that the finest relatedness information is required to accurately estimate $V_{A}$ and $h^{2}$. But this view $(i)$ increases experimental costs and (ii) overlooks that finest relatedness information is estimated with higher uncertainties.

The lattest methological developments in quantitative genetics aimed at using molecular markers through multilocus association models derived from genomic selection studies (Visscher, 2009; Yang et al., 2010). In this approach, the quality of $V_{A}$ and $h^{2}$ estimates depends on the linkage disequilibrium between the genotyped loci and the causal loci (Yang et al., 2010) and therefore requires genome wise high-density markers. Such genetic information is becoming reachable for a number of species thanks to next generation sequencing, but remains unaccessible for typical studies on non-model species. In addition, the genomic heritability and the trait heritability parameters are not equivalent and are equal only when all causal variants are typed (de los Campos et al., 2015), which remains very challenging for most species. While statistical methods derived from genomic selection offer promising prospects for estimating quantitative genetics parameters in natural populations (Robinson et al., 2013), there is still an interest for methods improving the estimation of $h^{2}$ by using a small number of informative markers to better infer relatedness in sibling groups for non-model organisms and notably plant species.

Here, we aimed at providing clear guidelines about the level at which the relateness information should be considered (pairwise-individual, family- or population-average) and about the

This article is protected by copyright. All rights reserved. 
model that should be used (family or animal model) according to different ecological scenarios.

We notably investigated different strategies to get the best information from only a subset of individuals genotyped at a limited number of loci. To that purpose, we used simulations and considered several departures from the half-sib assumptions potentially challenging the performance of the methods. More specifically, we considered three ecologically realistic scenarios: (i) unequal male fecundities and preferential mating, (ii) inbreeding and (iii) maternal effects on the simulated trait. In a first set of simulations, we used the relatedness data derived from multi-generational pedigrees and the one-generation pedigrees without paternity errors to test the "theoretical" validity of the methods. Then, we tested the sensitivity of the methods to the use of realistic genetic data, i.e. relatedness coefficients estimated from microsatellite markers and pedigrees with paternity errors.

\section{Material and method}

\section{SimUlation MODEL}

We used the model described in Gay et al. (2013) to simulate populations evolving at constant population size for a number $N_{T}$ of non-overlapping generations. We started from 200 diploid and unrelated hermaphrodite individuals at generation 0 with $L_{M}$ neutral unlinked loci of 10 alleles each and 500 unlinked Quantitative Trait Loci (QTL) of 5 alleles each. The allele frequencies at all loci evolved under random drift and without mutation, selection nor migration. Microsatellite markers can perform equally well as SNPs to assign paternity (Weinman et al., 2015). Therefore, we only considered microsatellites in our simulations and gave priority to test a large number of scenarios rather than different types of markers. The phenotypic value of 
an individual $k$ from family $j$ was computed as:

$$
P_{j, k}=\sum_{l}\left(v_{j, k, l, i}+v_{j, k, l, i^{\prime}}\right)+\varepsilon_{j, k}
$$

with $v_{j, k, l, i}$ and $v_{j, k, l, i^{\prime}}$ the allelic effects at the QTL $l$ of the alleles $i$ and $i^{\prime}$ of the offspring $k$ in the family $j$, randomly drawn from a normal distribution $\left(N\left(0, \sigma_{v}^{2}\right)\right)$, and $\varepsilon_{k}$ the environmental effect, drawn from a normal distribution $\left(N\left(0, \sigma_{\varepsilon}^{2}\right)\right)$.

We modified this model to account for non-random mating, ancestral inbreeding and maternal effects (Table 1). At the last generation (generation 1 or 3 here), we sampled 15 maternal progenies and phenotyped 40 offspring in each, reaching a total of 600 offspring. We assumed that only a subset of $N_{g}=15$ offspring per family have a known one-generation pedigree and genotype at the neutral markers. For all simulated scenarios (except scenario 0), we aimed at simulating biologically realistic relatedness structures, i.e. non-negligible nor excessive average paternal relatednesses between offspring. We adjusted the parameters $\alpha$ and $N_{P C}$ accordingly (see below).

\section{Non-random mating}

We considered two origins of non-random mating. First, males can unevenly contribute to the reproduction. We modeled individual fecundity for each male $p\left(F_{p}\right)$ using a Dirichlet distribution, with a scale parameter $\alpha$ :

$$
\left(F_{1}, \ldots, F_{p}, \ldots, F_{N p o p}\right) \sim D\left(\alpha ; \frac{1}{N p o p}, \ldots, \frac{1}{N p o p}\right)
$$

Low values of $\alpha(\alpha<1)$ generate unequal fecundities, i.e. few highly fecund individuals and many individuals with low fecundities. We considered either equal male fecundities $(\alpha=5$, 
scenario 0$)$ or unequal male fecundities $(\alpha=0.01$, scenario $i$; Table 1$)$.

Non-random mating can also result from spatial or temporal differentiation of male gamete pools, e.g. due to preferential mating. The mothers of the last generation were split into $N_{P C}$ groups of mothers that share the same pollen pool. We generated as many distributions of male fecundities as the number of pollen pools $\left(N_{P C}\right)$. The differentiation of pollen pools increases with $N_{P C}\left(N_{P C}=1\right.$ : a single pollen pool; $N_{P C}=15$ : pollen pools highly differentiated $)$. In scenario $i$ with unequal male fecundities, we examined $N_{P C}=\{1,5,15\}$. In this scenario, no selfing occured $(s=0)$ to avoid confusion between the effects of selfing and paternal sibship sizes on the increase of relatedness.

\section{Ancestral inbreeding}

For each mating event, selfing could occur with probability $s$. In scenario $i i$ ancestral inbreeding was considered by increasing the number of generations $\left(N_{T}=3\right)$ and allowing selfing with probabilities $s=\{0,0.2,0.4\}$. In this scenario we simulated slighter unequal fecundities $(\alpha$ $=0.1$ and $\left.N_{P C}=5\right)$ than in the other scenarios to avoid generating excessive and unrealistic paternal relatednesses within families.

\section{Maternal effects}

In scenario $i i i$ we included maternal effects on the offspring phenotypes as non-transgenerational effects (i.e. effects did not affect the resemblance between offspring and their mother; de Villemereuil et al. 2013):

$$
P_{j, k}=\sum_{l}\left(v_{j, k, l, i}+v_{j, k, l, i^{\prime}}\right)+m_{j}+\varepsilon_{j, k}
$$

This article is protected by copyright. All rights reserved. 
with $m_{j}$ the maternal effect of the mother $j$ on the phenotype of its offspring $k$. The maternal effects were normally distributed, $m_{j} \sim N\left(0, \sigma_{m}^{2}\right)$, and $\sigma_{m}^{2}=\{0,0.22,0.32\}$ (Table 1$)$. Scenario $i i i$ also simulated different paternal sibship sizes $\left(\alpha=0.01\right.$ and $N_{P C}=5$; Table 1$)$.

\section{Estimation of genetic relatednesses}

In all simulations, we recorded the multi-generational pedigree to calculate the relatedness coefficient $\rho_{k, k^{\prime}}$ between each pair of individuals $\left(k, k^{\prime}\right)$ and the one-generation pedigree $\rho_{k, k^{\prime}}^{(1)}$. In a first set of simulations, the performance of the methods for estimating quantitative genetic parameters (described below) were compared under the 4 different scenarios, assuming that the relatednesses were known without errors (i.e. $\rho_{k, k^{\prime}}$ and $\rho_{k, k^{\prime}}^{(1)}$ ) for the $N_{g}$ genotyped offspring.

Then, we examined the performances when using realistic relatedness information. To that purpose, we first generated the one-generation pedigree with $20 \%$ of paternity error simulated randomly, $\widetilde{\rho}_{k, k^{\prime}}^{(1)}$. We then used the similarities at molecular markers between pairs of genotyped individuals to estimate pairwise individual relatedness coefficients using the approach of Loiselle et al. (1995). This estimator performs well in presence of departures from HardyWeinberg equilibrium and rare alleles (Gay et al., 2013). While this approach is usually used on the diploid genotypes, here we chose to apply it on the haploid paternal contributions retrieved from the genotypes of the offspring (as proposed by Ritland 2002 to investigate the correlation of paternity) to take advantage of the known maternal relatedness relationships. We thus estimated the paternal relatednesses between each pair of genotyped offspring $\left(k, k^{\prime}\right), \widetilde{\rho}_{p\left(k, k^{\prime}\right)}$. Negative $\widetilde{\rho}_{p\left(k, k^{\prime}\right)}$ were replaced by zero following Gay et al. (2013). The total pairwise relatedness was then calculated as : $\widetilde{\rho}_{k, k^{\prime}}=\widetilde{\rho}_{m\left(k, k^{\prime}\right)}+\widetilde{\rho}_{p\left(k, k^{\prime}\right)}$, with $\widetilde{\rho}_{m\left(k, k^{\prime}\right)}$, the average maternal 
relatedness equal to 0.25 or 0 for offspring from the same or different families respectively (i.e. no use of the molecular markers for the maternal part). These assumptions about maternal relatedness likely hold for most of the progeny designs, except for natural populations with high inbreeding, where the relatedness between mothers can be non-null. In this second set of simulations, we simulated non-random mating (scenario $i$ with $N_{P C}=5$ and $\alpha=0.01$ ) with a number of neutral markers $L_{M}=\{10 ; 20 ; 40\}$ to test the effect of paternity errors and variable numbers of microsatellite loci on the performance of the methods. Note that the number of loci did not affect the paternity errors, mimicking the paternity analyses which control the rate of false paternity (Marshall et al., 1998).

All the scenarios were simulated with a moderate value of heritability $\left(h^{2}=0.33\right)$. The simulation program was written in $\mathrm{C}++$ and run in batch using a python script.

\section{ESTIMATION OF HERITABILITY}

Based on the simulated data sets, we estimated $V_{A}$ and heritability $h^{2}=\frac{V_{A}}{V_{P}}\left(V_{P}\right.$, the total phenotypic variance). We report here the $h^{2}$ estimates and their deviation to the true value. We used 7 main methods ( 6 tested methods +1 method as a reference) differing by the model (either the family or animal model) and the relatedness information used (categorical or continuous, pairwise or averaged; Table 2). All the methods tested used the same amount of genetic data.

\section{Family model}

This article is protected by copyright. All rights reserved. 
We used a family model accounting only for maternal relatedness:

$$
P_{j, k}=\mu+\text { Fam }_{j}+\varepsilon_{j, k}
$$

where $P_{j, k}$ is the phenotype of offspring $k$ from family $j, \mu$ is the mean, Fam $_{j}$ a random family effect with variance $V_{F}$ and $\varepsilon_{j, k}$ a residual error with variance $V_{R}$.

In the simplest "Family" method, $h^{2}$ was estimated as (Falconer \& MacKay, 1996):

$$
h_{\text {Family }}^{2}=\frac{4 V_{F}}{V_{F}+V_{R}}
$$

Then, following Squillace (1974), we corrected this estimate using the mean genetic relatedness estimated within families $\rho_{w}$, to account for $\rho_{w}>0.25$ in sibling designs ("FamAdj" method):

$$
h_{\text {FamAdj }}^{2}=\frac{\frac{1}{\rho_{w}} V_{F}}{V_{F}+V_{R}}
$$

We used another correction inspired from Squillace (1974) to account for non-null relatedness among families in natural populations ("FamDoubleAdj" method):

$$
h_{\text {FamDoubleAdj }}^{2}=\frac{V_{F}}{\left(\rho_{w}-\rho_{b}\right) V_{R}+\rho_{w} . V_{F}}
$$

where $\rho_{b}$ is the mean genetic relatedness estimated between families.

$\rho_{w}$ and $\rho_{b}$ were computed by averaging $\rho_{k, k^{\prime}}\left(\right.$ or $\left.\widetilde{\rho}_{k, k^{\prime}}\right)$ over all pairs of individuals $\left(k, k^{\prime}\right)$ with a known molecular information. These models were fitted in R using the package "lme4".

\section{Animal model}

This article is protected by copyright. All rights reserved. 
For this second set of methods, we estimated the additive genetic variance $\left(V_{A}\right)$ in the framework of the animal model:

$$
P_{j, k}=\mu+a_{j, k}+\varepsilon_{j, k}
$$

with $\mu$ the mean, $a_{j, k}$ the random additive genetic effect for individual $k$ in family $j$ and $\varepsilon_{j, k}$ a residual error. A variance component was added to account for maternal effects in scenario iii:

$$
P_{j, k}=\mu+a_{j, k}+m_{j}+\varepsilon_{j, k}
$$

with $m_{j}$ the random effect of mother $j$. In both models, the additive genetic effects $a_{j, k}$ were normally distributed with mean 0 and a variance-covariance matrix, $G=V_{A} A$, where $A$ is the additive genetic relationship matrix with individual elements $\rho_{k, k^{\prime}}$.

We filled the matrix $A$ following four different methods:

- The "PhiA" matrix contained the pairwise relatedness coefficients calculated from the multigenerational pedigree $\left(\rho_{k, k^{\prime}}\right)$ for all of the phenotyped individuals. This matrix was used as a reference to evaluate the loss of performance of the other methods, since it exploits the most accurate knowledge of pairwise relatednesses, generally unknown in experimental studies. Similarly, all the pairwise $\widetilde{\rho}_{k, k^{\prime}}$ estimated with the Loiselle approach were used to test the classical "pedigree-free" approach (Gay et al., 2013), in the "PhiA ${\tilde{\widetilde{\rho}} k k^{\prime}}$ " matrix.

- The "Relatedness" matrix, inspired from Bush et al. (2011), estimates average relatedness coefficients at the family level from the pairwise $\rho_{k, k^{\prime}}$ (or $\widetilde{\rho}_{k, k^{\prime}}$ ) recorded for the $N_{g}$ individuals per family with a known molecular information.

This article is protected by copyright. All rights reserved. 


$$
\begin{gathered}
\rho_{w, j}=\sum_{k, k^{\prime} \in \operatorname{family}(j)} \frac{\rho_{k, k^{\prime}}}{N g_{j}\left(N g_{j}-1\right) / 2} \\
\rho_{b, j j^{\prime}}=\sum_{k \in j} \sum_{k^{\prime} \in j^{\prime}} \frac{\rho_{k, k^{\prime}}}{N g_{j} N g_{j}^{\prime}}
\end{gathered}
$$

with $N g_{j}$ the number of genotyped offspring in family $j$.

The $A$ matrix contains the $\rho_{w, j}$ (or $\widetilde{\rho}_{w, j}$ if estimated with realistic data sets) for each family $j$ (instead of 0.25 ) and the $\rho_{b, j j^{\prime}}$ (or $\left.\widetilde{\rho}_{b, j j^{\prime}}\right)$ for each family pair $\left(j, j^{\prime}\right)$ (instead of 0 ; Figure $1 \mathrm{~b}$ ). This method relates to the "FamDoubleAdj" method, but uses the animal model framework and accounts for different average relatednesses between families.

- The "HS+FS" matrix, inspired from El-Kassaby et al. (2011), combines the classic half-sib assumptions with the pairwise relatednesses estimated using the one-generation pedigree $\left(\rho_{k, k^{\prime}}^{(1)}\right.$ or $\widetilde{\rho}_{k, k^{\prime}}^{(1)}$ ) for the subset of genotyped individuals (assuming that all the genotyped offspring can be assigned to a father). Individuals can thus be unrelated $\left(\rho_{k, k^{\prime}}=0\right)$, HS $\left(\rho_{k, k^{\prime}}=0.25\right)$ or FS $\left(\rho_{k, k^{\prime}}=0.5 ;\right.$ Figure 1c).

- The "Hybrid" matrix mixes the relatedness information from the "HS+FS" and "Relatedness" matrices to optimally combine all the information contained in the genetic data. As in "HS+FS", pairwise relatedness between the genotyped offspring $\left(\rho_{k, k^{\prime}}^{(1)}\right.$ or $\left.\widetilde{\rho}_{k, k^{\prime}}^{(1)}\right)$ was estimated using the one-generation pedigree (with or without errors; Figure 1d). In addition, the molecular data available from this subset was also used to estimate the average genetic relatedness coefficients within $\left(\rho_{w, j}\right.$ or $\left.\widetilde{\rho}_{w, j}\right)$ and between families $\left(\rho_{b, j j^{\prime}}\right.$ or $\left.\widetilde{\rho}_{b, j j^{\prime}}\right)$. These family-average relatedness coefficients are used instead of the family assumptions for the non-genotyped individuals (Figure 1d). With realistic data sets, we also tested the replacement of the pedigree information by the pairwise $\widetilde{\rho}_{k, k^{\prime}}$ coefficients estimated from microsatellite markers for the $N_{g}$ genotyped individuals. This second hybrid matrix was called "Hybrid $\widetilde{\rho}_{k, k^{\prime}}$ ". While the other 
methods are derived from previous publications, the "Hybrid" approach is new.

The "Relatedness" and "Hybrid $\widetilde{\rho}_{k, k^{\prime}}$ " methods are pedigree-free animal models. The $A$ matrices with non-categorical relatednesses can be non-positive definite (Frentiu et al., 2008). Rather than forcing the inversion of $A$ in the animal model, we derived $\bar{A}$, a positive definite matrix, close to $A$, by modifying the eigenvalues following the method described in the supplementary material (appendix A). The animal models were fitted using the $\bar{A}$ matrix in AsReml v3.0.5 (Gilmour et al., 2006).

\section{Performance indices}

The different methods were tested on $n=50$ replicated simulations per parameter set. We used three performance indices applied to $h^{2}:(1)$ the relative bias $b(\hat{\theta})$, defined as $\left(\frac{1}{n} \sum_{i=1}^{n} \frac{\left(\hat{\theta}_{i}-\theta_{i}\right)}{\theta_{i}}\right) x 100$ (in $\%$ ), where $\hat{\theta}$ is the estimator and $\theta$ the true parameter value, (2) the mean square error $\operatorname{MSE}(\hat{\boldsymbol{\theta}})=\frac{1}{n} \sum_{i=1}^{n}\left(\hat{\theta}_{i}-\theta_{i}\right)^{2}$ and (3) the coverage, corresponding to the proportion of simulations where the $95 \%$ confidence intervals of $\hat{\theta}$ contained $\theta$. Confidence intervals of the estimates were computed from the approximate standard errors (s.e.) estimated by AsReml, assuming a normal distribution $\left(h^{2} \pm 1.96\right.$ s.e. $)$. As there is no standard method to estimate s.e. using the family model framework, the coverage index were only computed for the methods using the animal model. The $95 \%$ confidence intervals of the bias $\left(C I_{b(\hat{\theta})}\right)$ were also estimated as twice the standard error of the 50 simulated $h^{2}$ estimates. The bias was thus considered significant if $b(\hat{\theta})-C I_{b(\hat{\theta})}>0$ or $b(\hat{\theta})+C I_{b(\hat{\theta})}<0$.

This article is protected by copyright. All rights reserved. 


\section{Results}

\section{NULL SCENARIO}

In presence of equal male fecundities (scenario 0, Table 1), the family model assumptions were met, i.e. the average relatedness within families was equal to the expected relatedness between HS and there was no relatedness between families $\left(\rho_{w}=0.25\right.$ and $\left.\rho_{b}=0.004\right)$. All the methods tested had low negative biases, not significantly different from zero (Table 3 ). The methods exploiting average relatedness information (both with the family and animal model) had lower performances than methods exploiting individual relatedness ("HS+FS" and "Hybrid" methods) both for bias and MSE (Table 3).

\section{EFFECT OF NON-RANDOM MATING (SCENARIO $i$ )}

As expected, non-random mating generated non-negligible paternal relatedness within and between families: $\rho_{w}$ rose to 0.34 and $\rho_{b}$ decreased from 0.09 to 0.003 with increasing differentiation of the pollen pools $\left(N_{P C}\right.$; Table 4$)$. The "Fam" method over-estimated $h^{2}$ when pollen pools were highly differentiated (for $N_{P C}=15, M S E_{\text {Fam }}=0.027$ and $b\left(h_{\text {Fam }}^{2}\right)=28$ ), because the family assumption underestimated the actual $\rho_{w}\left(\rho_{w}=0.33\right.$ instead of 0.25$)$. However, the "Family" method was no more biased in absence of pollen pool differenciation $\left(N_{P C}=1\right.$, $M S E_{\text {Fam }}=0.013$ and $\left.b\left(h_{\text {Fam }}^{2}\right)=-5 \%\right)$. In this case, $\rho_{w}$ and $\rho_{b}$ were similarly inflated $\left(\rho_{w}=\right.$ 0.34 instead of 0.25 and $\rho_{b}=0.089$ instead of 0 ) and the errors generated by underestimating the relatednesses within and between families counterbalanced each other. Thus, in presence of unequal male fecundities, the performance of the simple family method decreased with pollen pool differentiation. In contrast, the bias of the "FamAdj" method increased with decreasing pollen pool differenciation $\left(b\left(h_{\text {FamAdj }}^{2}\right)=-29.5 \%\right.$ for $\left.N_{P C}=1\right)$ since this method corrected 
for the inflated $\rho_{w}$ but not for $\rho_{b}$. The MSE and biases of the "FamDoubleAdj" method were low for all values of $N_{P C}\left(M S E_{\text {FamDoubleAdj }}<0.017, b\left(h_{\text {FamDoubleAdj }}^{2}\right)<8 \%\right)$ and allowed to efficiently adjust $h^{2}$ values for the different departures from $\rho_{w}=0.25$ and $\rho_{b}=0$.

Methods based on the animal model performed well regardless of the level of pollen pool differentiation, with low bias and MSE (Table 4). All the methods tended to under-estimate $h^{2}$ when $\rho_{w}$ and $\rho_{b}$ were inflated. For the simulated sample design (600 offspring sampled from 15 mothers) the inclusion of $37 \%$ of paternal information (15 offspring per family) seemed sufficient for an accurate estimation of $h^{2}$ in presence of different paternal sibship sizes.

\section{EFFECT OF ANCESTRAL INBREEDING (SCENARIO $i$ i)}

Ancestral inbreeding largely increased the mean genetic relatedness within families, with $\rho_{w}$ $>0.40$ (Table 4) instead of $\rho_{w}=0.25$ under the HS assumption (Table 5). With only 3 generations simulated, inbreeding did not affect relatedness between families ( $\rho_{b}=0.016$ with $s$ $=0$ and $\rho_{b}=0.017$ with $s=0.2$ ). Inbreeding decreased the performance of the classic family model $\left(M S E_{\text {Fam }}>0.053\right.$ and $\left.b\left(h_{\text {Fam }}^{2}\right)>49 \%\right)$. The adjustment of the family model using $\rho_{w}$ and/or $\rho_{b}$ corrected those biases $\left(b\left(h_{\text {FamAdj }}^{2}\right)>-13 \%\right.$ and $\left.b\left(h_{\text {FamDoubleAdj }}^{2}\right)<12 \%\right)$, but the "FamAdj" method appeared more efficient than the "FamDoubleAdj" method in this particular case $\left(M S E_{\text {FamAdj }} \in[0.012 ; 0.017]\right.$ and $\left.M S E_{\text {FamDoubleAd } j} \in[0.021 ; 0.028]\right)$.

Methods using the animal model and including average relatedness information ranging over several generations ("Relatedness" and "Hybrid") outperformed the methods restricted to one-generation ("FS+HS", $M S E_{F S+H S}>0.023$ ). Yet, strong inbreeding tended to decrease the 
coverage power and increase the bias of all methods (for "Relatedness" and "Hybrid": bias became significant and $\operatorname{cov} \in[0.76 ; 0.82]$ for $s=0.40)$.

\section{EFFECT OF MATERNAL COMPONENTS (SCENARIO iii)}

Maternal effects decreased the performance of all methods as compared to the "PhiA" reference: MSE were always larger than 0.028 and biases were all significantly different from zero (Table 6). As expected, with increasing maternal effects, the family model was inappropriate compared to the animal model with a random maternal effect $\left(M S E_{\text {Family }} \in[0.086 ; 0.238]\right.$ and $M S E_{\text {animal }} \in[0.035 ; 0.075]$ for $\left.V_{M}=0.08 V_{P}\right)$. While family models tended to overestimate $h^{2}$, animal models tended to underestimate $h^{2}$.

Among the methods using the animal model, the "Relatedness" method, which only uses average relatedness within and between families, performed worst: the coverage was reduced and the MSE was higher in presence of maternal effects $\left(M S E_{\text {Relatedness }}=0.067\right.$ and cov $_{\text {Relatedness }}$ $=0.56$ for $V_{M}=0.04 V_{P}$ ). Finally, with maternal effects, the proportion of paternal information added by genotyping (37\% here) was not sufficient to accurately estimate $h^{2}\left(b\left(h_{F S+H S}^{2}\right)<-25\right.$ $\left.\%, b\left(h_{\text {Hyrid }}^{2}\right)<-21 \%\right)$. Note that even if all offspring had a known pedigree ("PhiA" method), the $h^{2}$ estimate would still be slightly biased $\left(b\left(h_{P h i A}^{2}\right)=-11.60 \pm 11.16\right.$ for $V_{M}=0.04$ and $b\left(h_{P h i A}^{2}\right)=-15.36 \pm 8.90$ for $V_{M}=0.08$; Table 6).

\section{EFFECT OF MOLECULAR MARKER UNCERTAINTY}

Adding $20 \%$ of pedigree errors did not decrease the performance of the "HS+FS" and "Hy-

This article is protected by copyright. All rights reserved. 
brid" methods (Table 7). The use of mean genetic relatedness information based on Loiselle coefficients $\widetilde{\rho}_{k, k^{\prime}}$ instead of the true $\rho_{k, k^{\prime}}$ did not decrease the performance of methods using average marker-based relatedness coefficients, i.e. "FamAdj", "FamDoubleAdj", "Relatedness" or "Hybrid" methods (Table 4 and 7). Only the methods using pairwise marker-based relatedness coefficients, i.e. "PhiA ${\widetilde{\rho} k k^{\prime}}^{\prime \prime}$ and "Hybrid $\widetilde{\rho}_{k, k^{\prime}}$, were affected by the use of realistic data sets (high biais and low coverage; Table 4 and 7). Note that counter-intuitively these methods presented low MSE (Table 7) which happens for estimators with large bias but very low variance (and generally very low coverage).

Interestingly, the amount of genotyped loci only slightly affected the methods using average marker-based coefficients (Table 7). At the opposite, methods using pairwise marker-based relatednesses showed, as expected, increasing performances with the amount of genotyped mark$\operatorname{ers}\left(b\left(h_{P h i A \widetilde{\rho} k k^{\prime}}^{2}\right)=-42 \%\right.$ and $\operatorname{cov}_{P h i A \widetilde{\rho} k k^{\prime}}=0.34$ for $N l o c i=10 ; b\left(h_{P h i A \widetilde{\rho} k k^{\prime}}^{2}\right)=-29$ and $\operatorname{cov}_{P h i A \widetilde{\rho} k k^{\prime}}$ $=0.70$ for Nloci $=40$; Table 7). Yet, even with 40 loci, methods using pairwise marker-based coefficients performed worse than methods using average marker-based coefficients with 10 loci.

\section{Discussion}

Progeny designs are relevant to study local adaptation and the adaptive potential of plant populations (Leimu \& Fischer, 2008; Hansen et al., 2012). Many studies advocate for a better use of long-term data from progeny trials for long-lifetime species, such as trees, for which a long history of provenance tests exists (Savolainen et al., 2007; Alberto et al., 2013). Although the 
major recent methodological developments focus on dense genetic markers maps to estimate $h^{2}$ using multilocus association models (Yang et al., 2010) or genomic relatednesses in animal models (Berenos et al., 2014), these approaches have recently been criticised (de los Campos et al., 2015). Therefore, there are still needs to improve the "standard" family or animal models to analyse these experiments at reasonable genotyping costs, notably for non-model species. Here, we used simulations to provide guidelines to make the best use of polymorphic molecular data.

\section{WRONG ADJUSTEMENT OF THE FAMILY MODEL CAN BIAS $h^{2}$ ESTIMATES IN PRES-} ENCE OF POLLEN POOL STRUCTURE

In plant natural populations, spatial or temporal structuration of pollen pools is common, due to restricted gamete dispersal and asynchronous reproductive phenologies (Hardy et al., 2004; de Lucas et al., 2008), leading to increased paternal relatedness between maternal families $\left(\rho_{b}\right)$. Yet, most empirical studies assume that $\rho_{b}$ is negligible and efforts have been focused on correcting for the departure from the assumption $\rho_{w}=0.25$ (or $\rho_{w}=0.5$ for FS families), using the "FamAdj" correction (Ramirez-Valiente et al., 2009).

Contrary to this common practice, our simulations showed that the "FamDoubleAdj" method, that accounts for both $\rho_{w}$ and $\rho_{b}$, was the most robust correction of the family model regarding the different departures from random mating (i.e. unequal fecundities and pollen pool structuration). In an extreme case where the males equally pollinated each female (i.e. only one pollen pool), we showed that the simple family model was no more biased (even in presence of uneven male fecundities). Squillace (1974) similarly showed that, when the parents of the 
progeny design were related (increasing $\rho_{b}$ ), the bias generated by wrong HS assumptions became mostly insignificant. Overall, insufficient corrections of $V_{A}$ estimates (i.e. according to $\rho_{w}$ only) may have led to systematically underestimate the adaptive potential of natural populations (underestimating the evolvability, $I_{A}$; Houle 1992) or to anticonservative tests of the action of natural selection on trait differentiation (overestimating $Q s t$ ).

\section{AVERAGE MARKER-BASED RELATEDNESS COEFFICIENTS EFFICIENTLY CORRECT}

\section{FOR INFLATED RELATEDNESSES IN PROGENY DESIGNS}

A critical question in quantative genetics is how to use molecular marker information to improve relatedness information (Thomas, 2005; Csillery et al., 2006; Gay et al., 2013; Wang, 2016). While the benefit of estimating $h^{2}$ using pairwise genomic relatednesses (estimated from high density SNP arrays; Wang 2016) and pedigree-free methods is increasingly recognized (Berenos et al., 2014), the guidelines about how exploiting microsatellite markers are still muddled. Consistently however, several authors (i) have shown that estimates of pairwise relatedness based on a small number of polymorphic markers suffer from large MSE and high sampling variance and thus (ii) argue in favour of pedigree reconstruction (Thomas, 2005; Csillery et al., 2006).

Here, we confirmed the difficulty to accurately estimate $h^{2}$ using pairwise relatedness $\widetilde{\rho}_{k k^{\prime}}$ coefficients and pedigree-free methods based on a reduced set of markers (PhiA $\mathrm{A}_{\widetilde{\rho} k k^{\prime}}$ and Hybrid $\widetilde{\rho}_{k, k^{\prime}}$ methods presented the lowest performances whatever the amount of genotyped markers; with $10 \leq N_{\text {loci }} \leq 40$ ). However, contrary to the statement $(i i)$, we showed that, for traits with simple determinism (scenarios $0, \mathrm{i}$, ii), the three pedigree-free methods using average markerbased relatedness coefficients (with the family model: "FamAdj", "FamDoubleAdj" and animal 
model: "Relatedness") performed as well as methods using pedigree information ("HS+FS" method) in presence of non-random mating and inbreeding. Interestingly, these methods were also far less sensitive than the methods using pairwise $\widetilde{\rho}_{k k^{\prime}}$ to the number of marker used. A practical conclusion is that microsatellite markers can improve relatedness information as well by using a simple averaging procedure as by using more assumption-dependent methods (i.e. pedigree reconstruction methods often require a priori knowledge about the number of generations to reconstruct, demographic history of the population; Cros et al. 2014). This may encourage using pedigree-free approaches with average marker-based coefficients. More specifically, we also confirmed that the use of average information calculated at the family level (in the "Relatedness" method) rather than at the population level (in the "FamAdj" and "FamDoubleAdj" methods) only slightly improved $h^{2}$ estimates (Bush et al., 2011). Overall, considering traits with simple determinism, fine-scale relatedness information (i.e. between individuals) is not required to accurately estimate $V_{A}$ and $h^{2}$ in sibling designs.

\section{CAN OPEN-POLLINATED DESIGNS BE USED TO INVESTIGATE COMPLEX TRAIT DE-}

\section{TERMINISM?}

Maternal effects affect many traits and species (Mousseau \& Fox, 1998; Raesaenen \& Kruuk, 2007) and can explain a substantial proportion of the total phenotypic variance (Wilson et al., 2005; Raesaenen \& Kruuk, 2007); they are thus non-negligible sources of bias when estimating $h^{2}$ in sibling designs. In presence of maternal effects, the use of individual pairwise relatedness information ("HS+FS" and "Hybrid" methods) improved the accuracy of the analysis of progeny designs compared to the use of average relatedness ("Relatedness" method). In animal models including a random term for maternal effects, the use of incomplete paternal relatedness

This article is protected by copyright. All rights reserved. 
information led to underestimate $h^{2}$. A similar trend was also observed by de Villemereuil et al. (2013, appendix D). More generally, if relatedness matrices do not contain enough information on paternal relationships, maternal and additive genetic variance estimates are biased due to confusion between covariance structures.

While El-Kassaby et al. (2011) showed that, in presence of non-random mating, the genotyping effort to improve the $h^{2}$ estimates in sibling groups can be optimised, in presence of maternal effects we showed that even considering the whole one-generation pedigree information, was insufficient to correctly estimate $h^{2}$. This result highlights that the additive genetic and maternal effects can only be properly disentangled if there are enough father-related offspring within OP families (depending on the population mating system and the sampling strategy). The amount of father-related offspring can therefore be a main limiting factor to study traits with complex genetic determinisms in natural populations.

\section{GUIDELINES TO ANALYZE PROGENY DESIGNS IN NON-MODEL SPECIES}

Our ecologically realistic scenarios showed the benefits and pitfalls of methods using either incomplete one-generation pedigree or average relatedness information: none appeared as a good solution to deal with every departure from random mating and the presence of environmental maternal effects. The new hybrid method proposed here always performed as well as the best of its two "parent" methods ("HS+FS" or "Relatedness") and was robust to the use of "realistic" genetic data sets (pedigrees with paternity errors and Loiselle marker-based coefficients). This method has the advantage of using the whole genetic information available when only a subset of individuals have been genotyped, gathering the one-generation pedigree and average marker- 
based coefficients. Its main drawback is that the $A$ matrix can be non-invertible. We proposed a modification of the matrix that solved the problem adequately (without this approximation the biases ranged from -10 to $-55 \%$ in scenario i). Other methods have been suggested to deal with non-inversible matrices, which is a classical issue for pedigree-free methods (Frentiu et al., 2008; Ahlinder \& Sillanpaa, 2013). Because of its robustness to various ecological scenarios, we suggest to use the "Hybrid" approach in sibling quantitative genetic designs for populations where no a priori knowledge exists about the mating system or the determinism of the traits.

More specifically, focusing on microsatellite markers, we confirmed that they were inefficient to estimate pairwise individual genetic relatednesses but they can still be reliably used to estimate average paternal relatedness coefficients. For traits with simple determinism and in presence of non-negligible paternal relatedness within and between maternal families, two main outcomes can be highlighted: (1) the use of fine relatedness information (i.e. at individual-level) through a pedigree instead of average relatedness information at the population- or family-level only brings low benefits on $h^{2}$ estimates. Therefore, methods using average marker-based coefficients, i.e. the "FamDoubleAdj" method for the family model and the "Relatedness" method for the animal model, have the best cost-efficiency ratio in these simple cases: they only require to genotype a set of representative offspring per family and their mother. However, we expect this conclusion to be challenged using genome wise high-density markers to estimate the "realised relatedness" between individuals. (2) As noticed by El-Kassaby et al. (2011) and this study, the efficiency gained by genotyping all offspring was clearly lower than the gain from genotyping only a representative subset of them (here about $40 \%$ ), whatever if this genotyping is used to reconstruct a pedigree or estimate average relatedness coefficients. A practical con-

This article is protected by copyright. All rights reserved. 
clusion is that moderate genotyping effort combined with pedigree-free approaches are simple and efficient to re-analyze existing sibling designs in order to account for non-random mating. The optimal genotyping effort is a case-by-case parameter depending on the family structure, the population mating system and the determinism of the trait. However, we do not expect the type of marker used (SNPs, microsatellite) to affect the optimal genotyping effort, as it only affects the accuracy of the relatedness estimates and not the representativity of the population relatedness structure. At the opposite, in presence of maternal environmental determinism, only methods using relatedness information at the individual level (i.e. "HS+FS" or "Hybrid" methods) were efficient to disentangle additive and maternal effects. In that case, increasing the amount of genotyped offspring will improve the $V_{A}$ and $h^{2}$ estimates only if the sampled families contain a high proportion of father-related offspring.

The approaches presented here are not restricted to plants and should also be relevant for animal species characterised by high fecundities (e.g. insects, fish species), for which relatedness coefficients estimated from a subset of offspring can provide relevant information about the relatedness structure within and between families. In animal studies, controlled crosses are generally easier to perform, or, at least, the set of reproductive adults generating the progeny population can be limited. It is thus usually simpler to reconstruct one-generation pedigrees in controlled experiments. But a challenge for these studies is now to make quantative genetics in unmanipulated natural populations to estimate in situ heritabilities (Zajitschek \& Bonduriansky, 2014). While next generation sequencing and genomic approaches offer promising prospects, the methods compared here to improve $V_{A}$ and $h^{2}$ estimates using cost-efficient genotyping should also participate to the growth of quantitative genetics in the wild for species without

This article is protected by copyright. All rights reserved. 
high density genetic data.

\section{Acknowledgements}

We are grateful to F. Lefèvre, M. Siol, K. Csillery and A. Bontemps for the discussion and comments on the study and the previous versions of this manuscript. We also thank the people of the GDR "Quantitative Genetics in Natural Populations" for the discussions about these issues and the reviewers for their comments that greatly improved the clarity of the manuscript. This study was funded by the ERANet BiodivERsA TipTree project (ANR-12-EBID-0003), part of the 2012 BiodivERsA call for research proposal, the metaprogramme Adaptation of Agriculture and Forests to Climate Change (AAFCC) of the French National Institute for Agricultural Research (INRA) and the MECC project (ANR-13-ADAP-0006).

This article is protected by copyright. All rights reserved. 


\section{References}

Ahlinder, J. \& Sillanpaa, M.J. (2013) Rapid Bayesian inference of heritability in animal models without convergence problems. Methods In Ecology And Evolution, 4, 1037-1046.

Alberto, F.J., Aitken, S.N., Alia, R., Gonzalez-Martinez, S.C., Hanninen, H., Kremer, A., Lefevre, F., Lenormand, T., Yeaman, S., Whetten, R. \& Savolainen, O. (2013) Potential for evolutionary responses to climate change evidence from tree populations. Global CHange Biology, 19, 1645-1661.

Ashley, M.V. (2010) Plant parentage, pollination, and dispersal: How dna microsatellites have altered the landscape. Critical Reviews in Plant Sciences, 29, 148-161.

Berenos, C., Ellis, P.A., Pilkington, J.G. \& Pemberton, J.M. (2014) Estimating quantitative genetic parameters in wild populations: a comparison of pedigree and genomic approaches. Molecular Ecology, 23, 3434-3451.

Blouin, M. (2003) DNA-based methods for pedigree reconstruction and kinship analysis in natural populations. Trends in Ecology and Evolution, 18, 503-511.

Bush, D., Kain, D., Matheson, C. \& Kanowski, P. (2011) Marker-based adjustment of the additive relationship matrix for estimation of genetic parameters-an example using eucalyptus cladocalyx. Tree Genetics and Genome, 7, 23-35.

Cros, D., Sanchez, L., Cochard, B., Samper, P., Denis, M., Bouvet, J.M. \& Fernandez, J. (2014) Estimation of genealogical coancestry in plant species using a pedigree reconstruction algorithm and application to an oil palm breeding population. Theoretical And Applied Genetics, 127, 981-994.

Csillery, K., Johnson, T., Beraldi, D., Clutton-Brock, T., Coltman, D., Hansson, B., Spong, G. \& Pemberton, J.M. (2006) Performance of marker-based relatedness estimators in natural populations of outbred vertebrates. Genetics, 173, 2091-2101.

de los Campos, G., Sorensen, D. \& Gianola, D. (2015) Genomic Heritability: What Is It? Plos Genetics, 11.

de Lucas, A.I., Robledo-Arnuncio, J.J., Hidalgo, E. \& González-Martínez, S.C. (2008) Mating system and pollen gene flow in mediterranean maritime pine. Heredity, 100, 390-399.

de Villemereuil, P., Gimenez, O. \& Doligez, B. (2013) Comparing parent-offspring regression with frequentist and Bayesian animal models to estimate heritability in wild populations: a simulation study for Gaussian and binary traits. Methods in Ecology \& Evolution, 4, 260275.

El-Kassaby, Y.A., Cappa, E.P., Liewlaksaneeyanawin, C., Klapste, J. \& Lstiburek, M. (2011) Breeding without breeding: is a complete pedigree necessary for efficient breeding? Plos One, 6, e25737.

Falconer, D. \& MacKay, T. (1996) Introduction to Quantitative Genetcis. Ed 4. Longmans Greenn, Harlow, Essex, UK. 
Fernandez, J. \& Toro, M. (2006) A new method to estimate relatedness from molecular markers. Molecular Ecology, 15, 1657-1667.

Frentiu, F.D., Clegg, S.M., Chittock, J., Burke, T., Blows, M.W. \& Owens, I.P.F. (2008) Pedigree-free animal models: the relatedness matrix reloaded. Proceedings of the Royal Society B-Biological Sciences, 275, 639-647.

Gauzere, J., Klein, E.K. \& Oddou-Muratorio, S. (2013a) Ecological determinants of mating system within and between three fagus sylvatica populations along an elevational gradient. Molecular Ecology, 22, 5001-5015.

Gauzere, J., Oddou-Muratorio, S., Pichot, C., Lefèvre, F. \& Klein, E. (2013b) Biases in quantitative genetic analyses using open-pollinated progeny tests from natural tree populations. Acta Botanica Gallica, 160 (3-4), 227-238.

Gay, L., Siol, M. \& Ronfort, J. (2013) Pedigree-free estimates of heritability in the wild: Promising prospects for selfing populations. Plos One, $\mathbf{8}$.

Gilmour, A., Gogel, B., Cullis, B. \& Thompson, R. (2006) Asreml User Guide Release 2.0. Hemel Hempsted: VSN International Ltd.

Hadfield, J.D., Richardson, D.S. \& Burke, T. (2006) Towards unbiased parentage assignment: combining genetic, behavioural and spatial data in a Bayesian framework. Molecular Ecology, 15, 3715-3730.

Hansen, M.M., Olivieri, I., Waller, D.M., Nielsen, E.E. \& Grp, G.W. (2012) Monitoring adaptive genetic responses to environmental change. Molecular Ecology, 21, 1311-1329.

Hardy, O.J., González-Martínez, S.C., Fréville, H., Boquien, G., Mignot, A., Colas, B. \& Olivieri, I. (2004) Fine-scale genetic structure and gene dispersal in centaurea corymbosa (asteraceae) i. pattern of pollen dispersal. Journal of Evolutionary Biology, 17, 795-806.

Henderson, C.R. (1984) Applications of Linear Models in Animal Breeding. University of Guelph, Guelph, Canada.

Hill, W.G. \& Kirkpatrick, M. (2010) What Animal Breeding Has Taught Us about Evolution. Futuyma, DJ and Shafer, HB and Simberloff, D, ed., Annual Review of Ecology, Evolution, and Systematics, volume 41, pp. 1-19.

Houle, D. (1992) Comparing Evolvability And Variability Of Quantitative Traits. Genetics, 130, 195-204.

Kang, K., Bila, A., Harju, A. \& Lindgren, D. (2003) Estimation of fertility variation in forest tree population. Forestry, 76 (3), 329-344.

Kruuk, L.E.B. (2004) Estimating genetic parameters in natural populations using the "animal model". Proceedings of the Royal Society Series B-Biological Sciences, 359, 873-890.

Leimu, R. \& Fischer, M. (2008) A Meta-Analysis of Local Adaptation in Plants. Plos One, 3. 
Loiselle, B., Sork, V.L., Nason, J.D. \& Graham, C. (1995) Spatial genetic structure of a tropical understory shrub, psychotria officinalis (rubiaceae). American Journal of Botany, 82, 14201425.

Marshall, T., Slate, J., Kruuk, L. \& Pemberton, J. (1998) Statistical confidence for likelihoodbased paternity inference in natural populations. Molecular Ecology, 7, 639-655.

Merilä, J. \& Crnokrak, P. (2001) Comparison of genetic differentiation at marker loci and quantitative traits. Journal of Evolutionary Biology, 14, 892-903.

Mousseau, T.A. \& Fox, C.W. (1998) The adaptive significance of maternal effects. Trends in Ecology and Evolution, 13, 403-407.

Pigliucci, M. (2008) Opinion - Is evolvability evolvable? Nature Reviews Genetics, 9, 75-82.

Raesaenen, K. \& Kruuk, L.E.B. (2007) Maternal effects and evolution at ecological time-scales. Functional Ecology, 21, 408-421.

Ramirez-Valiente, J.A., Lorenzo, Z., Soto, A., Valladares, F., Gil, L. \& Aranda, I. (2009) Elucidating the role of genetic drift and natural selection in cork oak differentiation regarding drought tolerance. Molecular Ecology, 18, 3803-3815.

Ritland, K. (2002) Extensions of models for the estimation of mating systems using $n$ independent loci. Heredity, 88, 221-228.

Roach, D. \& Wulff, R. (1987) Maternal effects in plants. Annual Review of Ecology Evolution and Systematics, 18, 209-235.

Robinson, M.R., Santure, A.W., DeCauwer, I., Sheldon, B.C. \& Slate, J. (2013) Partitioning of genetic variation across the genome using multimarker methods in a wild bird population. Molecular Ecology, 22, 3963-3980.

Savolainen, O., Pyhajarvi, T. \& Knurr, T. (2007) Gene flow and local adaptation in trees. Annual Review of Ecology Evolution and Systematics, 38, 595-619.

Squillace, A. (1974) Average genetic correlations among offspring from open-pollinated forest trees. Silvae Genetica, 23, 149-156.

Stinchcombe, J. (2014) Chapter 8 Cross-pollination of palnts and animals: wild quantitative genetics and plant evolutionary genetics. In : Quantitative genetics in the wild. Oxford University Press.

Thomas, S. (2005) The estimation of genetic relationships using molecular markers and their efficiency in estimating heritability in natural populations. Philosophical Transactions Of The Royal Society B-biological Sciences, 360, 1457-1467.

Vekemans, X. \& Hardy, O. (2004) New insights from fine-scale spatial genetic structure analyses in plant populations. Molecular Ecology, 13, 921-935.

Visscher, P.M. (2009) Whole genome approaches to quantitative genetics. Genetica, 136, 351358. 3rd International Conference of Quantitative GENETICS, Zheijiang Univ, Hangzhou, PEOPLES R CHINA, AUG 18-24, 2007. 
Wang, J. (2016) Pedigrees or markers: Which are better in estimating relatedness and inbreeding coefficient? Theoretical Population Biology, 107, 4-13.

Ward, M., Dick, C.W., Gribel, R. \& Lowe, A.J. (2005) To self, or not to self... a review of outcrossing and pollen-mediated gene flow in neotropical trees. Heredity, 95, 246-254.

Weinman, L.R., Solomon, J.W. \& Rubenstein, D.R. (2015) A comparison of single nucleotide polymorphism and microsatellite markers for analysis of parentage and kinship in a cooperatively breeding bird. Molecular Ecology Resources, 15, 502-511.

Wilson, A., Coltman, D., Pemberton, J., Overall, A., Byrne, K. \& Kruuk, L. (2005) Maternal genetic effects set the potential for evolution in a free-living vertebrate population. Journal of Evolutionary Biology, 18, 405-414.

Yang, J., Benyamin, B., McEvoy, B.P., Gordon, S., Henders, A.K., Nyholt, D.R., Madden, P.A., Heath, A.C., Martin, N.G., Montgomery, G.W., Goddard, M.E. \& Visscher, P.M. (2010) Common SNPs explain a large proportion of the heritability for human height. Nature Genetics, 42, 565-U131.

Zajitschek, F. \& Bonduriansky, R. (2014) Chapter 9 Quantitative genetics of wild populations of arthropods. In : Quantitative genetics in the wild. Oxford University Press.

This article is protected by copyright. All rights reserved. 


\section{Data Accessibility}

Simulation code ( $\mathrm{C}++$, Python)and an example of AsReml script will be accessible from a Web hosting service developed by the BioSP laboratory. An example of the simulation outputs are available from Dryad Digital Repository: doi:10.5061/dryad.k25qf (provisional DOI).

\section{Author Contributions}

JG performed the analyses, wrote the first draft of the text and contributed to the revisions. LG provided the simulation code. LG and EKK modified the code with JG. JG, EKK, SOM and LG elaborated the simulation design. EKK, SOM and LG contributed to the successive revisions.

This article is protected by copyright. All rights reserved. 
Table 1: Summary of the parameters for the simulation scenarios investigating the effect of (i) unequal male reproductive success, (ii) ancestral inbreeding and (iii) maternal effects on $h^{2}$ estimates. Scenario 0 is the null scenario with random mating system.

\begin{tabular}{|c|l|c|c|c|c|}
\hline Parameter & Definition & Scenario 0 & Scenario $i$ & Scenario $i i$ & Scenario iii \\
\hline$N_{T}$ & Number of generations & 1 & 1 & 3 & 1 \\
\hline$\sigma_{v}$ & $\begin{array}{l}\text { Standard deviation of } \\
\text { the allelic effect }\end{array}$ & 0.02 & 0.02 & 0.02 & 0.02 \\
\hline$\sigma_{e}$ & $\begin{array}{l}\text { Standard deviation of } \\
\text { the environmental ef- } \\
\text { fect }\end{array}$ & 0.9 & 0.9 & 0.9 & $\{0.84,0.87\}$ \\
\hline$\sigma_{m}$ & $\begin{array}{l}\text { Standard deviation of } \\
\text { the maternal effect }\end{array}$ & 0 & 0 & 0 & $\{0.22,0.32\}$ \\
\hline$s$ & $\begin{array}{l}\text { Proportion of selfing } \\
\text { Desequilibrium in male } \\
\text { fecundities }\end{array}$ & 0 & 0 & $\{0,0.2,0.4\}$ & 0 \\
\hline$N_{P C}$ & $\begin{array}{l}\text { Number of maternal } \\
\text { pollen pools }\end{array}$ & 15 & $\{1,5,15\}$ & 5 & 0.01 \\
\hline
\end{tabular}

This article is protected by copyright. All rights reserved. 
Table 2: Relatedness information used in each method. Relatedness information is defined at two main scales: fine-scale, i.e. pairwise-individual, and broad-scale, i.e. family- or populationaverage. $\rho_{k, k^{\prime}}$ represents the pairwise individual marker-based relatedness coefficients.

\begin{tabular}{c|c|l|l}
\hline \multirow{3}{*}{ Model } & Method name & Individual level & Family or population level \\
\hline \multirow{2}{*}{ Family } & Family & - & HS assumptions \\
\cline { 2 - 4 } & FamAdj & - & $\begin{array}{l}\text { average } \rho_{k, k^{\prime}} \text { within families at the } \\
\text { population level }\end{array}$ \\
\cline { 2 - 5 } & FamDoubleAdj & - & $\begin{array}{l}\text { average } \rho_{k, k^{\prime}} \text { within and between } \\
\text { families at the population level }\end{array}$ \\
\hline Animal & Relatedness & - & $\begin{array}{l}\text { average } \rho_{k, k^{\prime}} \text { within and between } \\
\text { families at the family level }\end{array}$ \\
\cline { 2 - 5 } & HS+FS & one-generation pedigree & HS assumptions \\
\cline { 2 - 5 } & Hybrid & $\begin{array}{l}\text { one-generation pedigree } \\
\text { marker-based coefficients } \rho_{k, k^{\prime}} \text { for } \\
\text { the derived Hybrid } \widetilde{\rho}_{k, k^{\prime}} \text { method) }\end{array}$ & $\begin{array}{l}\text { average } \rho_{k, k^{\prime}} \text { within and between } \\
\text { families at the family level }\end{array}$ \\
\hline
\end{tabular}

This article is protected by copyright. All rights reserved. 
Table 3: Performance (relative bias in $\%$, MSE and coverage) of the methods to estimate $h^{2}$ under the null scenario $\left(N_{P C}=1, \alpha=5\right)$. The mean genetic relatedness within $\left(\rho_{w}\right)$ and among families $\left(\rho_{b}\right)$ is indicated.

\begin{tabular}{c|lcl}
\hline & \multicolumn{3}{|c}{ Scenario 0 } \\
Method & Bias \pm 2 s.e & MSE & Cov \\
\hline Family & $-7.26 \pm 14.81$ & 0.022 & - \\
FamAdj & $-7.83 \pm 14.70$ & 0.022 & - \\
FamDoubleAdj & $-6.45 \pm 14.93$ & 0.022 & - \\
Relatedness & $-6.30 \pm 15.02$ & 0.022 & 0.82 \\
HS+FS & $-5.91 \pm 13.51$ & 0.017 & 1 \\
Hybrid Matrix & $-4.99 \pm 13.55$ & 0.017 & 0.82 \\
PhiA & $-5.94 \pm 10.81$ & 0.011 & 1 \\
\hline$\rho_{w}$ & \multicolumn{3}{|c}{0.25} \\
$\rho_{b}$ & \multicolumn{3}{|c}{0.004} \\
\hline
\end{tabular}

This article is protected by copyright. All rights reserved. 


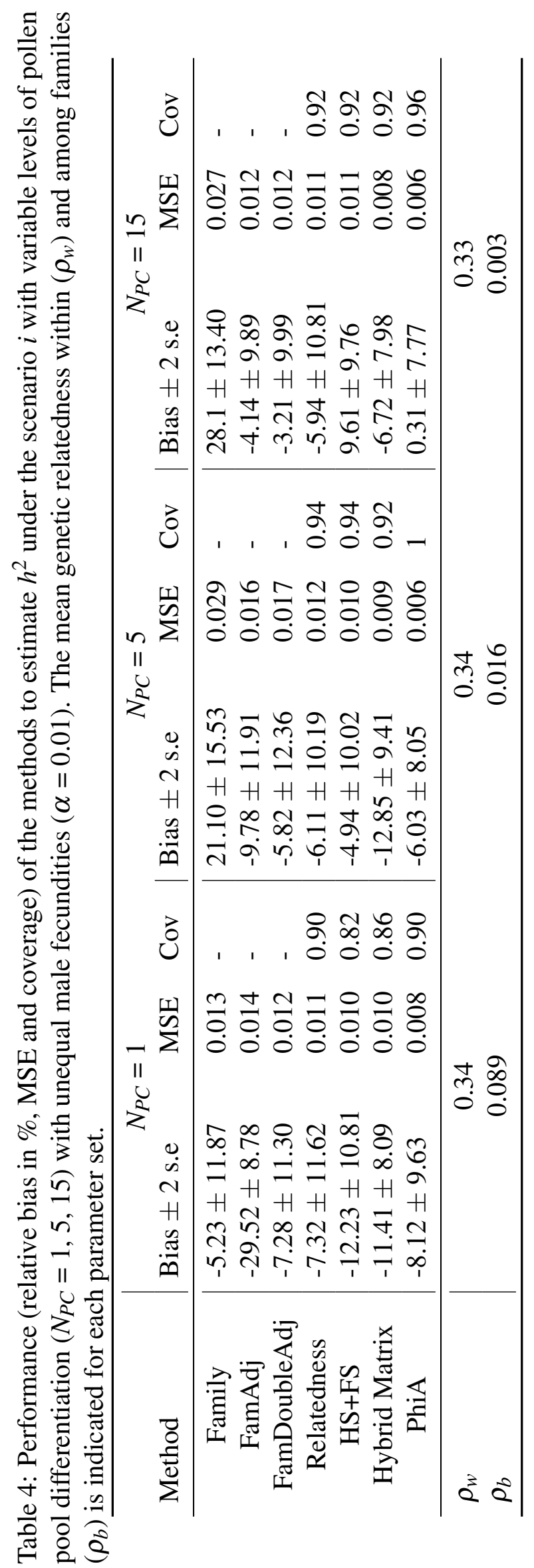

This article is protected by copyright. All rights reserved. 


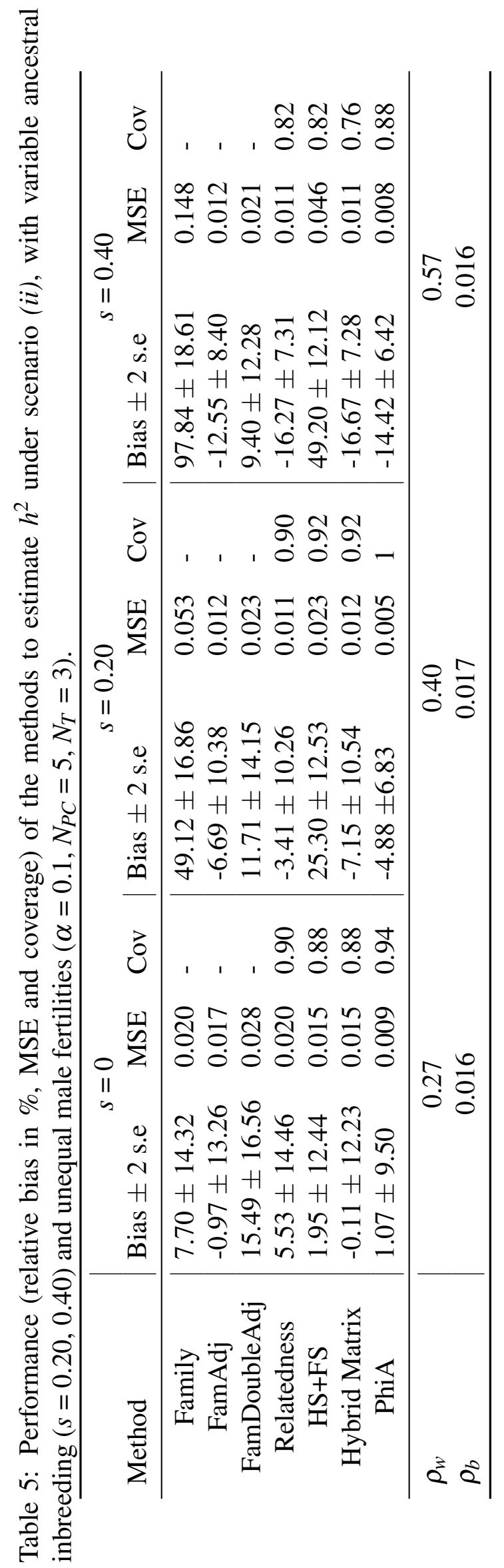

This article is protected by copyright. All rights reserved. 


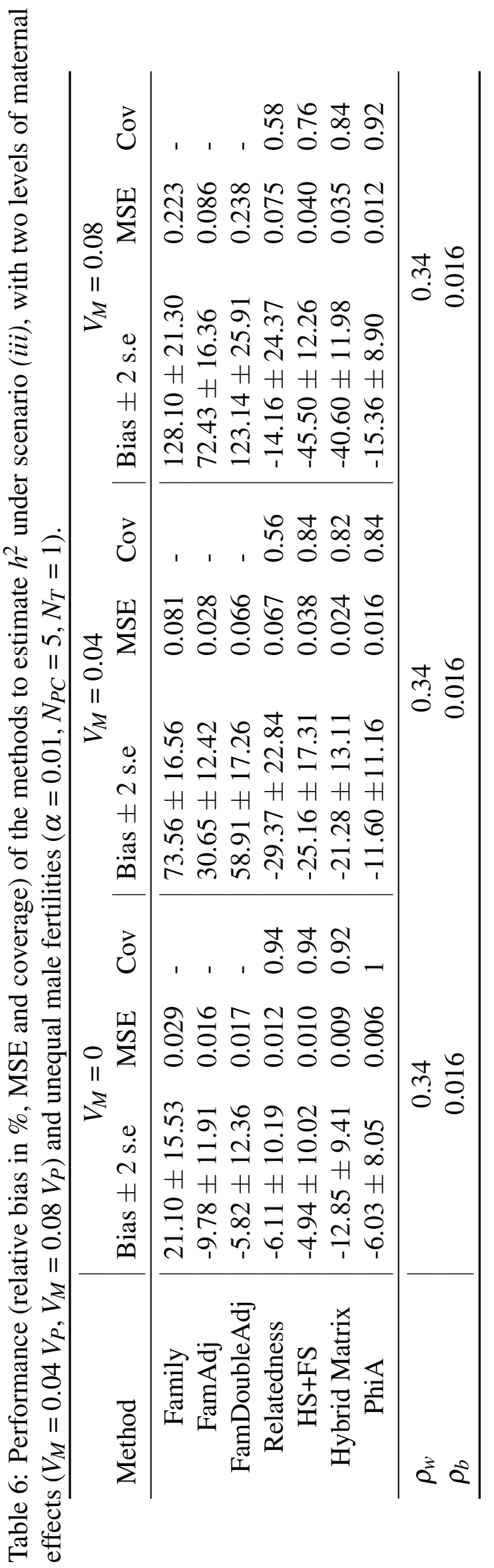

This article is protected by copyright. All rights reserved. 


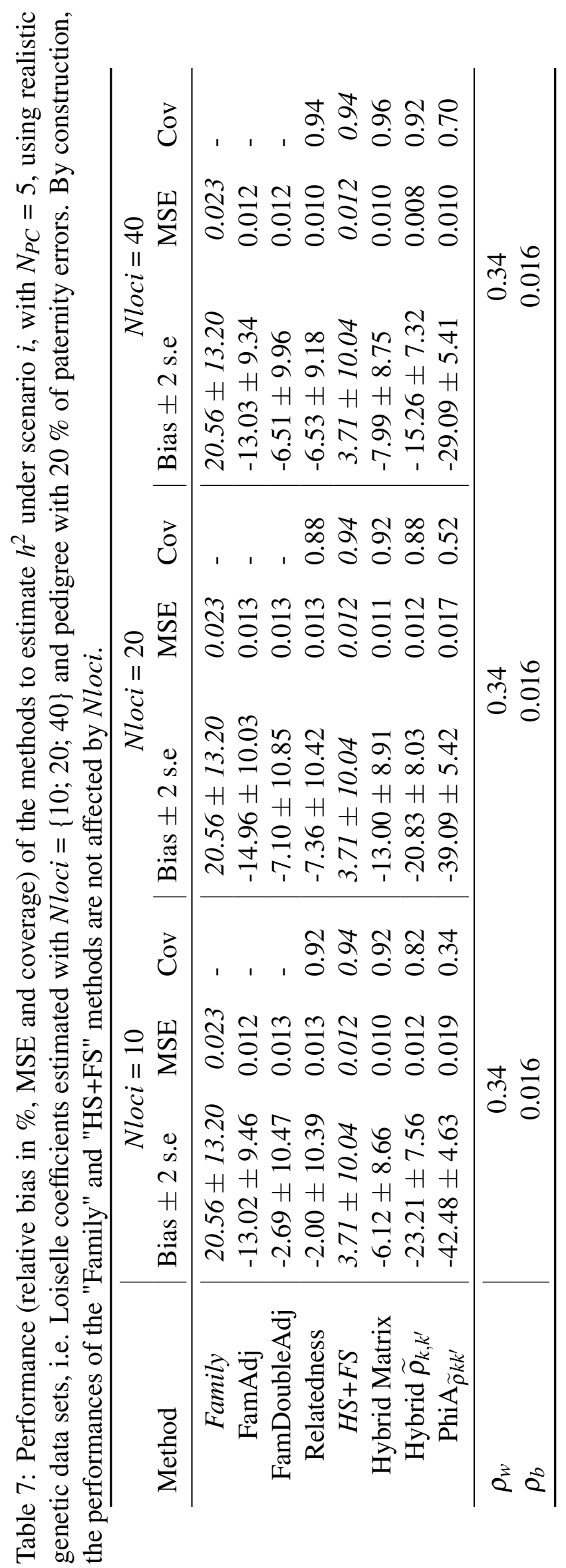

This article is protected by copyright. All rights reserved. 
Figure 1: (a) Pedigree structure and representation of the corresponding (b) "Relatedness", (c) "HS+FS" and (d) "Hybrid" matrices, with $N$ fam $=2$, Noff $=5$ and $N g=3$ (genotyped individuals are represented in bold).

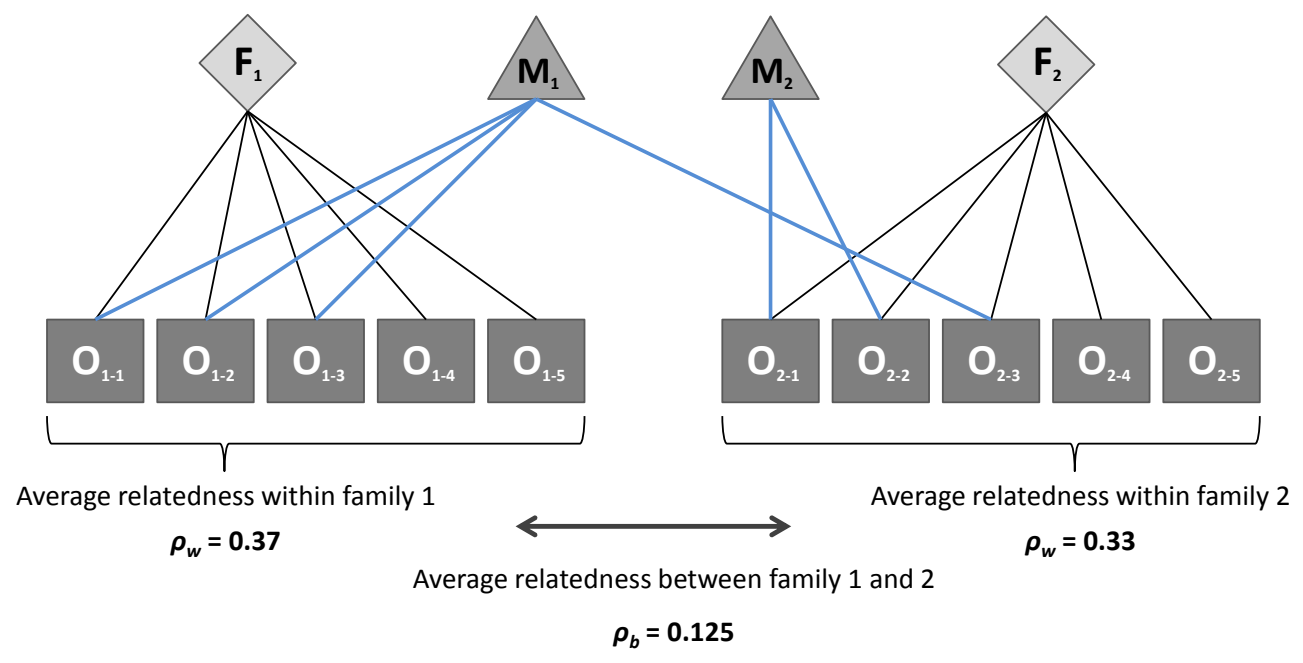

(a)

$\left(\begin{array}{ccccc|ccccc}\mathbf{1} & \mathbf{0 . 3 7} & \mathbf{0 . 3 7} & 0.37 & 0.37 & \mathbf{0 . 1 2 5} & \mathbf{0 . 1 2 5} & \mathbf{0 . 1 2 5} & 0.125 & 0.125 \\ \mathbf{0 . 3 7} & \mathbf{1} & \mathbf{0 . 3 7} & 0.37 & 0.37 & \mathbf{0 . 1 2 5} & \mathbf{0 . 1 2 5} & \mathbf{0 . 1 2 5} & 0.125 & 0.125 \\ \mathbf{0 . 3 7} & \mathbf{0 . 3 7} & \mathbf{1} & 0.37 & 0.37 & \mathbf{0 . 1 2 5} & \mathbf{0 . 1 2 5} & \mathbf{0 . 1 2 5} & 0.125 & 0.125 \\ 0.37 & 0.37 & 0.37 & 1 & 0.37 & 0.125 & 0.125 & 0.125 & 0.125 & 0.125 \\ 0.37 & 0.37 & 0.37 & 0.37 & 1 & 0.125 & 0.125 & 0.125 & 0.125 & 0.125 \\ \hline \mathbf{0 . 1 2 5} & \mathbf{0 . 1 2 5} & \mathbf{0 . 1 2 5} & 0.125 & 0.125 & \mathbf{1} & \mathbf{0 . 3 3} & \mathbf{0 . 3 3} & 0.33 & 0.33 \\ \mathbf{0 . 1 2 5} & \mathbf{0 . 1 2 5} & \mathbf{0 . 1 2 5} & 0.125 & 0.125 & \mathbf{0 . 3 3} & \mathbf{1} & \mathbf{0 . 3 3} & 0.33 & 0.33 \\ \mathbf{0 . 1 2 5} & \mathbf{0 . 1 2 5} & \mathbf{0 . 1 2 5} & 0.125 & 0.125 & \mathbf{0 . 3 3} & \mathbf{0 . 3 3} & \mathbf{1} & 0.33 & 0.33 \\ 0.125 & 0.125 & 0.125 & 0.125 & 0.125 & 0.33 & 0.33 & 0.33 & 1 & 0.33 \\ 0.125 & 0.125 & 0.125 & 0.125 & 0.125 & 0.33 & 0.33 & 0.33 & 0.33 & 1\end{array}\right)$

(b)

$$
\left(\begin{array}{ccccc|ccccc}
\mathbf{1} & \mathbf{0 . 5} & \mathbf{0 . 5} & 0.25 & 0.25 & \mathbf{0} & \mathbf{0} & \mathbf{0 . 2 5} & 0 & 0 \\
\mathbf{0 . 5} & \mathbf{1} & \mathbf{0 . 5} & 0.25 & 0.25 & \mathbf{0} & \mathbf{0} & \mathbf{0 . 2 5} & 0 & 0 \\
\mathbf{0 . 5} & \mathbf{0 . 5} & \mathbf{1} & 0.25 & 0.25 & \mathbf{0} & \mathbf{0} & \mathbf{0 . 2 5} & 0 & 0 \\
0.25 & 0.25 & 0.25 & 1 & 0.25 & 0 & 0 & 0 & 0 & 0 \\
0.25 & 0.25 & 0.25 & 0.25 & 1 & 0 & 0 & 0 & 0 & 0 \\
\hline \mathbf{0} & \mathbf{0} & \mathbf{0} & 0 & 0 & \mathbf{1} & \mathbf{0 . 5} & \mathbf{0 . 2 5} & 0.25 & 0.25 \\
\mathbf{0} & \mathbf{0} & \mathbf{0} & 0 & 0 & \mathbf{0 . 5} & \mathbf{1} & \mathbf{0 . 2 5} & 0.25 & 0.25 \\
\mathbf{0 2 5} & \mathbf{0 2 5} & \mathbf{0 . 2 5} & 0 & 0 & \mathbf{0 . 2 5} & \mathbf{0 . 2 5} & \mathbf{1} & 0.25 & 0.25 \\
0 & 0 & 0 & 0 & 0 & 0.25 & 0.25 & 0.25 & 1 & 0.25 \\
0 & 0 & 0 & 0 & 0 & 0.25 & 0.25 & 0.25 & 0.25 & 1
\end{array}\right)
$$

(c)

$\left(\begin{array}{ccccc|ccccc}\mathbf{1} & \mathbf{0 . 5} & \mathbf{0 . 5} & 0.37 & 0.37 & \mathbf{0} & \mathbf{0} & \mathbf{0 . 2 5} & 0.125 & 0.125 \\ \mathbf{0 . 5} & \mathbf{1} & \mathbf{0 . 5} & 0.37 & 0.37 & \mathbf{0} & \mathbf{0} & \mathbf{0 . 2 5} & 0.125 & 0.125 \\ \mathbf{0 . 5} & \mathbf{0 . 5} & \mathbf{1} & 0.37 & 0.37 & \mathbf{0} & \mathbf{0} & \mathbf{0 . 2 5} & 0.125 & 0.125 \\ 0.37 & 0.37 & 0.37 & 1 & 0.37 & 0.125 & 0.125 & 0.125 & 0.125 & 0.125 \\ 0.37 & 0.37 & 0.37 & 0.37 & 1 & 0.125 & 0.125 & 0.125 & 0.125 & 0.125 \\ \hline \mathbf{0} & \mathbf{0} & \mathbf{0} & 0.125 & 0.125 & \mathbf{1} & \mathbf{0 . 5} & \mathbf{0 . 2 5} & 0.33 & 0.33 \\ \mathbf{0} & \mathbf{0} & \mathbf{0} & 0.125 & 0.125 & \mathbf{0 . 5} & \mathbf{1} & \mathbf{0 . 2 5} & 0.33 & 0.33 \\ \mathbf{0 . 2 5} & \mathbf{0 . 2 5} & \mathbf{0 . 2 5} & 0.125 & 0.125 & \mathbf{0 . 2 5} & \mathbf{0 . 2 5} & \mathbf{1} & 0.33 & 0.33 \\ 0.125 & 0.125 & 0.125 & 0.125 & 0.125 & 0.33 & 0.33 & 0.33 & 1 & 0.33 \\ 0.125 & 0.125 & 0.125 & 0.125 & 0.125 & 0.33 & 0.33 & 0.33 & 0.33 & 1\end{array}\right)$

(d)

This article is protected by copyright. All rights reserved. 


\section{Appendix A: Algorithm used to substitute a definite-positive relatedness matrix in the animal model}

Filling a relatedness matrix $A$ with pairwise relatedness coefficients estimated from molecular markers (relying on Loiselle estimates for instance) frequently leads to a non definite-positive matrix, thus non-inversible (Frentiu et al. 2008). This was particularly frequent for the hybrid matrices investigated in the manuscript (e.g. for the "Hybrid" method).

For these problematic cases, AsReml proposes to use the "!NSD" command, which allows the matrix to be negative (semi)definite (Gilmour et al. 2006). But this solution does not provide robust estimates of the variance components: the heritability was largely underestimated (bias= $-44.52 \%,-19.87 \%,-11.06 \%$ for scenario $i$ and $N_{P C}=1,5,15$ respectively). Below we propose a modification of the relatedness matrix that makes it definite-positive.

Consider the symetric relatedness matrix $A$ of dimension $n \times n$ and the spectral decomposition of $A$ as

$$
A=U \Lambda U^{T}
$$

where $U$ is an upper triangular matrix of coefficients representing the eigenvectors and $\Lambda$ is the diagonal matrix containing the eigenvalues $\lambda_{1} \geq \ldots \geq \lambda_{n}$ of $A$. If $A$ is non definite-positive then there exist a $q$ such that $0<q<n$, the $n-q>0$ last eigenvalues are negative or null and $\lambda_{q}>0$.

We modify the matrix $\Lambda$ into a diagonal matrix $\bar{\Lambda}$ containing the following values $\overline{\lambda_{1}} \geq \ldots \geq \overline{\lambda_{n}}$ :

$$
\begin{gathered}
\overline{\lambda_{k}}=\lambda_{k}, \text { for } k \leq q \\
\overline{\lambda_{k}}=\lambda_{q} h^{k-q}, \text { for } k>q
\end{gathered}
$$

where $h<1$ controls the decrease of the modified $\bar{\lambda}$ 's towards 0 . A too small $h$ value can lead to numerical errors. We used $h=0.9$.

This correction means that the non-positive eigenvalues are replaced by a geometrically decreasing serie of strictly positive eigenvalues.

The modified relatedness matrix $\bar{A}$ is then obtained as

$$
\bar{A}=U \bar{\Lambda} U^{T}
$$

This matrix $\bar{A}$ was substituted for $A$ in the animal model analysed with AsReml. All matrix inversions of $\bar{A}$ 's were completed correctly by AsReml (no warning message).

This article is protected by copyright. All rights reserved. 